\title{
EFFECT OF ROUGHNESS TO HYDRAULIC PROJECTION IN AN U-SHAPED CHANNEL, WITH ROUGH BED
}

\author{
A. Ghomri*, M. Debabeche and F. Riguet
}

LARHYSS, Université de Biskra, BP 145 RP - 07000 Biskra, - Algérie

Received: 26 February 2011 / Accepted: 03 April 2010 / Published online: 30 June 2011

\begin{abstract}
This study aims to study the hydraulic jump controlled by threshold, moving in a channel profile ' $U$ ' bottomed rough for a single roughness. Functional relations in dimensionless terms, linking the different characteristics of the projection, showing the effect of roughness of the bottom of the channel are obtained.
\end{abstract}

Key words: Hydraulic jump, roughness, stilling basin, channel bottom rough.

\section{INTRODUCTION}

L'étude a pour but d'étudier l'effet d'une rugosité sur le ressaut hydraulique contrôlé par seuil en canal profilé en " $U$ " à fond rugueux pour une rugosité bien déterminée $\varepsilon=$ 9,11mm. L'étude bibliographique montre que les caractéristiques adimensionnelles essentielles, intervenant au phénomène du ressaut, dans un canal profilé en ' $U$ ', sont le nombre de Froude $\mathrm{IF}_{1}$ ou le débit relatif $\mathrm{Q}^{*}$, la hauteur relative amont $\mathrm{y}_{1}=\mathrm{h}_{1} / \mathrm{D}\left(\mathrm{h}_{1}\right.$ est la hauteur au pied du ressaut, $D$ est la largeur du canal $)$, la hauteur relative aval $y_{2}=h_{2} / D\left(h_{2}\right.$ est la hauteur maximale mesurée à l'aval du ressaut) et le rapport $\mathrm{y}=\mathrm{h}_{2} / \mathrm{h}_{1}$ des hauteurs conjuguées du ressaut.

L'objectif principal de cet article est de connaître l'effet de cette rugosité imposé au fond du canal profilé en " $U$ " aux caractéristiques du ressaut hydraulique.

Author Correspondence, e-mail: alighomri@yahoo.fr

ICID: 1020787 


\section{POSITION DU PROBLEME}

La modification des conditions à l'amont (hauteurs, débit,...etc) et à l'aval (type d'obstacle, sa position, sa hauteur, ...etc), peut conduire à différentes configurations de ressaut. Le ressaut est dit classique lorsqu'il se forme dans un canal rectangulaire de pente faible ou nulle, sans obstacle à l'aval. Il est dit contrôlé lorsque sa formation est conditionnée par la mise en place d'un obstacle à l'aval de l'écoulement.

Pour notre cas il s'agit d'un ressaut contrôlé par seuil mince en canal profilé en ' $U$ ' à fond demi-circulaire rugueux. Une rugosité équivalente a été étudiée $\varepsilon=9,11 \mathrm{~mm}$.

En effet, Pour une hauteur initiale $\mathrm{h}_{1}$ fixe, l'accroissement du débit entraîne à la fois le déplacement du ressaut vers l'aval et l'augmentation de sa longueur de rouleau $\mathrm{L}_{\mathrm{R}}$. $\mathrm{La}$ distance $\Delta \mathrm{x}$ sur laquelle le ressaut s'étend augmente également et pour ramener celui-ci dans sa position initiale, c'est à dire à environ $5 \mathrm{~cm}$ de la sortie du convergent, le premier seuil de hauteur s doit être surélevé. Ainsi à chaque valeur du débit relatif $\mathrm{Q}^{*}$, correspond une longueur $L_{j}$ du ressaut et $L r$ du rouleau, ainsi qu'une hauteur $h_{2}$ à l'aval du ressaut et une hauteur s du seuil (figure1).

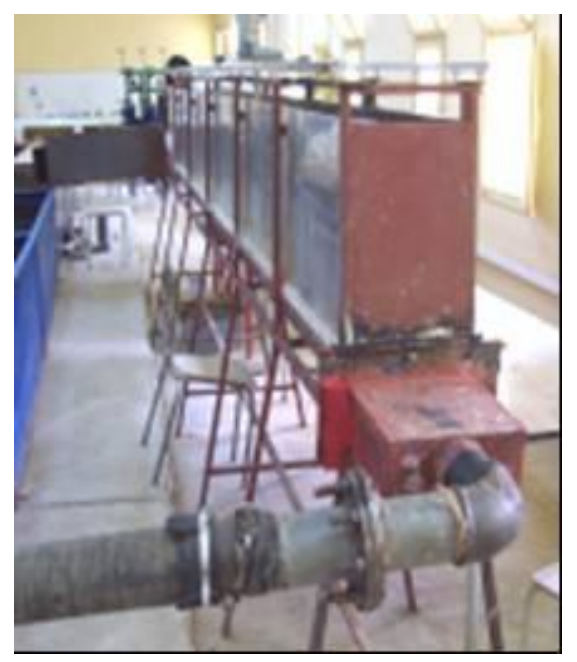

Photographie 1. Un banc d'expérimentation.

Ressaut contrôlé par seuil mince. $\mathrm{h}_{1}$ hauteur d'eau amont, $\mathrm{h}_{2}$ hauteur d'eau aval, $\mathrm{s}$ hauteur $\mathrm{du}$ seuil, $\mathrm{L}_{\mathrm{r}}$ longueur du rouleau de surface, $\mathrm{x}$ distance séparant la boite en charge $\mathrm{B}$ du pied du ressaut, B boite en charge. 


\section{PROTOCOLE EXPERIMENTAL}

\section{Description du modèle}

Le canal de mesure qui nous a servi de banc d'essai (photographie 1) est constitué essentiellement d'une rigole demi-circulaire en 'pvc' de 5,67 $\mathrm{m}$ de longueur, et de diamètre 24,5 cm sur laquelle est fixée latéralement, d'un coté une tôle en aluminium de $0,50 \mathrm{~m}$ de hauteur et de l'autre coté une série de 7 panneaux transparents en Plexiglas permettant les prises de vues et observations. Ces panneaux sont reliés entre eux par collage au chloroforme. Le tout est fixé par boulonnage à une structure métallique rigide. L'étanchéité est assurée par des joints en caoutchouc et par de la silicone.

Le fond du canal est parfaitement horizontal ou de pente nulle. Un bassin d'alimentation est relié au canal par le moyen d'une conduite circulaire de $100 \mathrm{~mm}$ de diamètre. Celle-ci est reliée à une boite métallique fermée, sur laquelle est insérée une ouverture à paroi plane en tôle de largeur déterminée débouchant dans le canal. Le rôle de cette paroi est de générer un écoulement incident à grande vitesse. La section de sortie de celle-ci est variable et sa hauteur correspondra à la hauteur initiale $\mathrm{h}_{1}$ du ressaut.

Le réglage des débits volumes s'effectue par manipulation de la vanne et sont mesurés à l'aide d'un débitmètre électromagnétique à affichage numérique.

L'alimentation du canal s'effectue par le moyen d'une pompe débitant jusqu'à 40 1/s.

\section{Description des essais}

L'expérimentation a été menée sous quatre hauteurs initiales $\left(\mathrm{h}_{1}(\mathrm{~cm})=2,3 ; 3,40 ; 5,2 ; 6,1\right)$. Une large gamme des nombres de Froude incidents a été ainsi obtenue $\left(2<\mathrm{IF}_{1}<15\right)$.

Des seuils de différentes hauteurs ont été testés, dont la hauteur s varie entre

\section{$3,4 \mathrm{~cm}$ et $19,8 \mathrm{~cm}$.}

Pour une hauteur $\mathrm{s}$ du seuil et pour une hauteur $\mathrm{h}_{1}$ de l'écoulement incident, l'augmentation du débit volume $\mathrm{Q}$, provoque l'apparition d'un ressaut (photographie 2.). Le couple de valeur $\left(\mathrm{Q}, \mathrm{h}_{1}\right)$ permet par ailleurs le calcul du débit relatif $\mathrm{Q}^{*}$. L'accroissement de $\mathrm{Q}^{*}$ entraîne à la fois le déplacement du ressaut vers l'aval et l'augmentation de sa longueur de ressaut $\mathrm{Lj}$. La distance $\Delta \mathrm{x}$ sur laquelle le ressaut s'étend augmente également et pour ramener celui-ci dans sa position initiale, c'est à dire à environ $4 \mathrm{~cm}$ à $5 \mathrm{~cm}$ de la sortie du 
convergent, le seuil de hauteur $\mathrm{s}$ doit être surélevé. Ainsi à chaque valeur du $\mathrm{Q}^{*}$ correspond une valeur de la longueur $\mathrm{Lj}$ de ressaut et une valeur de la hauteur s du seuil.

Un échantillon constitué d'une plusieurs de points de mesures expérimentales, pour chaque caractéristique, a permis ainsi d'aboutir à des résultats significatifs. Ces paramètres sont : la rugosité $\varepsilon$ du fond du canal, le débit $\mathrm{Q}$, la hauteur initiale $\mathrm{h}_{\mathrm{l}}$, la hauteur finale $\mathrm{h}_{2}$, la hauteur $\mathrm{s}$ du seuil, la longueur $\mathrm{Lj}$ du ressaut, compté à partir du début du ressaut.

Ceux-ci permettent de composer les produits adimensionnels suivants :

1 le débit relatif $\mathrm{Q}^{*}$ ou le nombre de Froude incident $\mathrm{IF}_{1}$,

$$
\begin{aligned}
& F_{1}=\sqrt{\frac{Q^{2} D \sin \alpha}{\left[\frac{D^{2}}{4}(\alpha-\sin \alpha \cos \alpha)\right]^{3}}} \\
& F_{1}=\frac{8 Q^{*} \sqrt{\sin \alpha}}{(\alpha-\sin \alpha \cos \alpha)^{3 / 2}} \\
& \alpha(r d)=\arccos (1-2 y 1) \\
& Q^{*}=\frac{Q}{\sqrt{g^{5}}}
\end{aligned}
$$

2 la hauteur relative amont $\mathrm{y}_{1}=\mathrm{h}_{1} / \mathrm{D}$,

3 la hauteur relative aval $\mathrm{y}_{2}=\mathrm{h}_{2} / \mathrm{D}$,

4 le rapport $\mathrm{y}=\mathrm{h}_{2} / \mathrm{h}_{1}$ des hauteurs conjuguées du ressaut.

5 la hauteur relative $S=s / h_{l}$ du seuil,

6 la longueur relative $\mathrm{Lj} / \mathrm{h}_{1}$ du ressaut,

7 la longueur relative $\mathrm{Lj} / \mathrm{h}_{2} \mathrm{du}$ ressaut.

8 la rugosité relative $\varepsilon / \mathrm{D}$. 
(a)

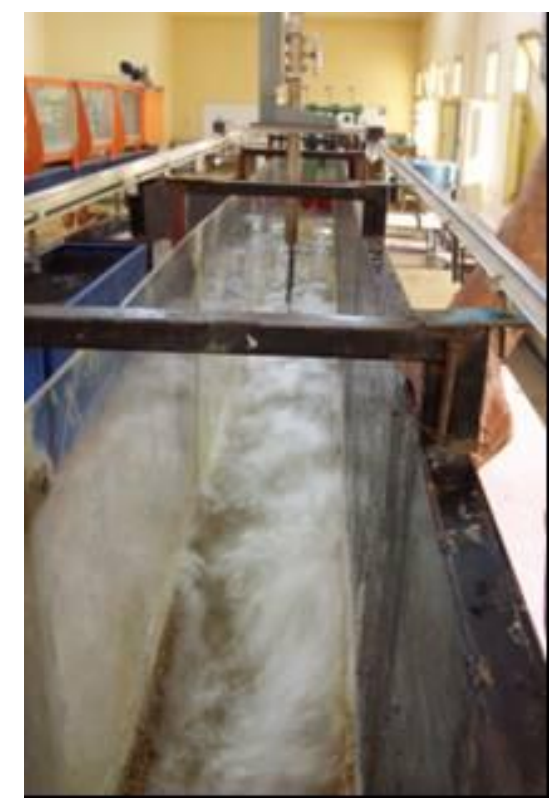

Photographie 2. Ressaut hydraulique évoluant dans un canal profilé en U à fond rugueux.

a) Vue à l'aval du ressaut

\section{Mode de préparation de la rugosité testée}

1 Le tamisage du gravier de granulométrie différente est effectué par le moyen d'un tamiseur électrique composé par plusieurs tamis de différent diamètres normalisés variant entre 3 et $12 \mathrm{~mm}$, avec des temps de tamisage bien fixés en relations avec la gamme de gravier à tamiser environ 5 min.

2 Après l'obtention de la gamme voulue, ce dernier est lavé puis séché.

3 Les gammes choisies, varient entre 3 et $12 \mathrm{~mm}$ selon le refus du tamis normalisé. Les particules obtenues sont ensuite uniformément réparties sur une toile en plastique

4 (photographie 3), puis collées par le moyen d'une colle Becta 10000 rigide.

5 Enfin, la rugosité équivalente obtenue est : $\varepsilon=9,11 \mathrm{~mm}$ 


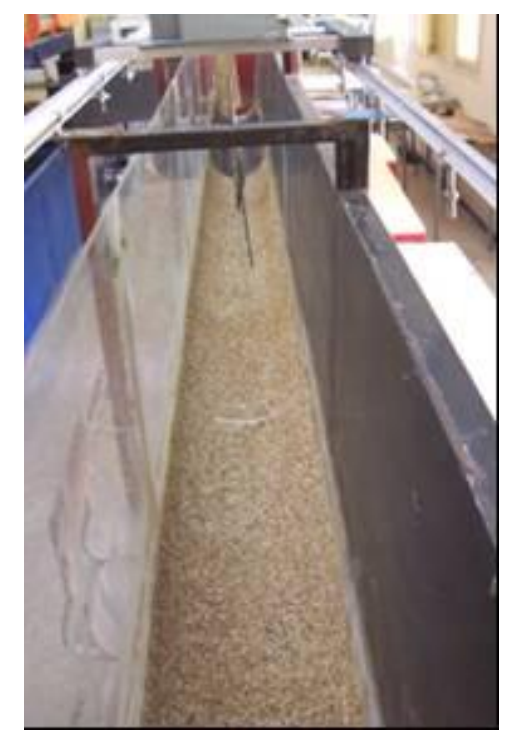

Photographie 3. Grains de gravier répartis uniformément sur le fond du canal.

\section{RESULTATS EXPERIMENTAUX}

\section{Hauteur relative aval $y_{2}$ en fonction du débit relatif $Q^{*}$}

La figure 1 représente la variation de la hauteur relative aval $\mathrm{y}_{2}$ en fonction du débit relatif $\mathrm{Q}^{*}$ du ressaut hydraulique contrôlé par seuil en canal à fond rugueux, de rugosité relative $\varepsilon / \mathrm{D}=0,037(\varepsilon=9,11 \mathrm{~mm})$, pour 04 valeurs distinctes de $\mathrm{y}_{1}$.

Quatre allures de points de mesures sont visibles, correspondant chacune à une hauteur relative fixe de $\mathrm{y}_{1}$. Les traits continus illustrent la courbe théorique issue de l'équation de quantité de mouvement pour les quatre valeurs de $\mathrm{y}_{1}: 0,0939 ; 0,1388 ; 0,2122 \mathrm{et} 0,2489$. 


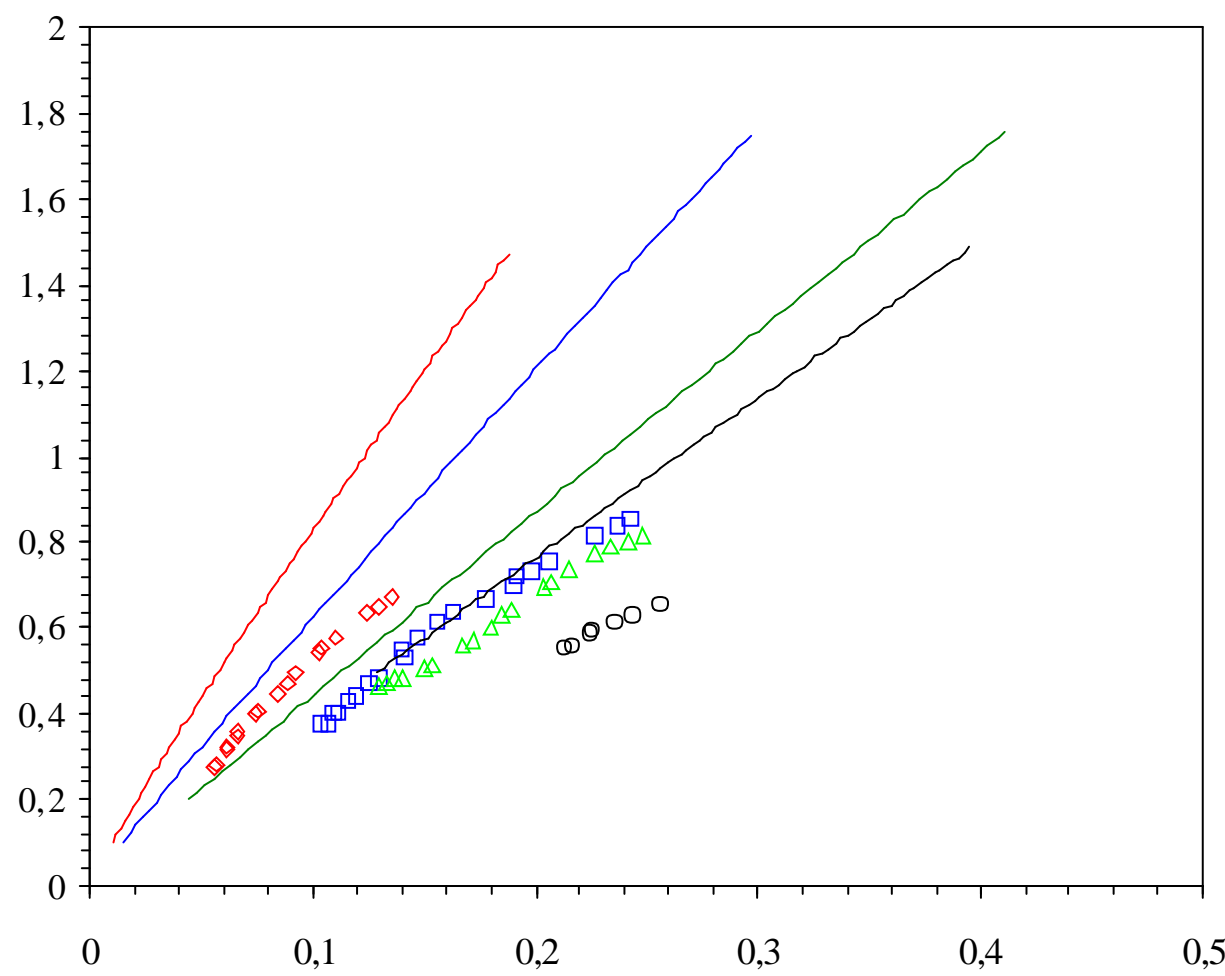

Fig.1. Ressaut hydraulique évoluant en canal à fond rugueux, de rugosité relative $\varepsilon / D=$ 0,037 variation de la hauteur relative aval y2 en fonction du débit relatif $Q^{*}$. Les motifs noirs vides représentent les points de mesures expérimentales. (-) Courbe théorique selon

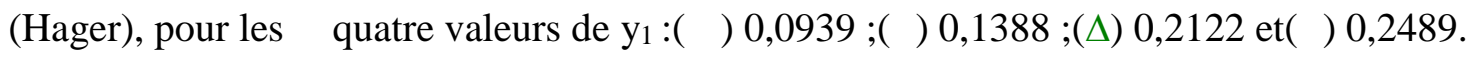

Cette figure montre que les points de mesures du ressaut contrôlé à fond rugueux se situent bien au-dessous des courbes théoriques (rugosité nulle).

En remarque en premier lieu que l'augmentation du débit relatif $Q^{*}$ entraîne celle de la hauteur relative aval. L'influence de la hauteur relative amont $\mathrm{y}_{1}$ est nettement perceptible. Par ailleurs, pour le même nombre débit relatif $\mathrm{Q}^{*}, \mathrm{y}_{2}$ diminue avec l'augmentation de $\mathrm{y}_{1}$.

La figure 2 montre quatre séries, correspondant chacune à une valeur bien connue de $\mathrm{y}_{1}$. Les traits continus représentent l'ajustement des points de mesures par la méthode des moindres carrés. 


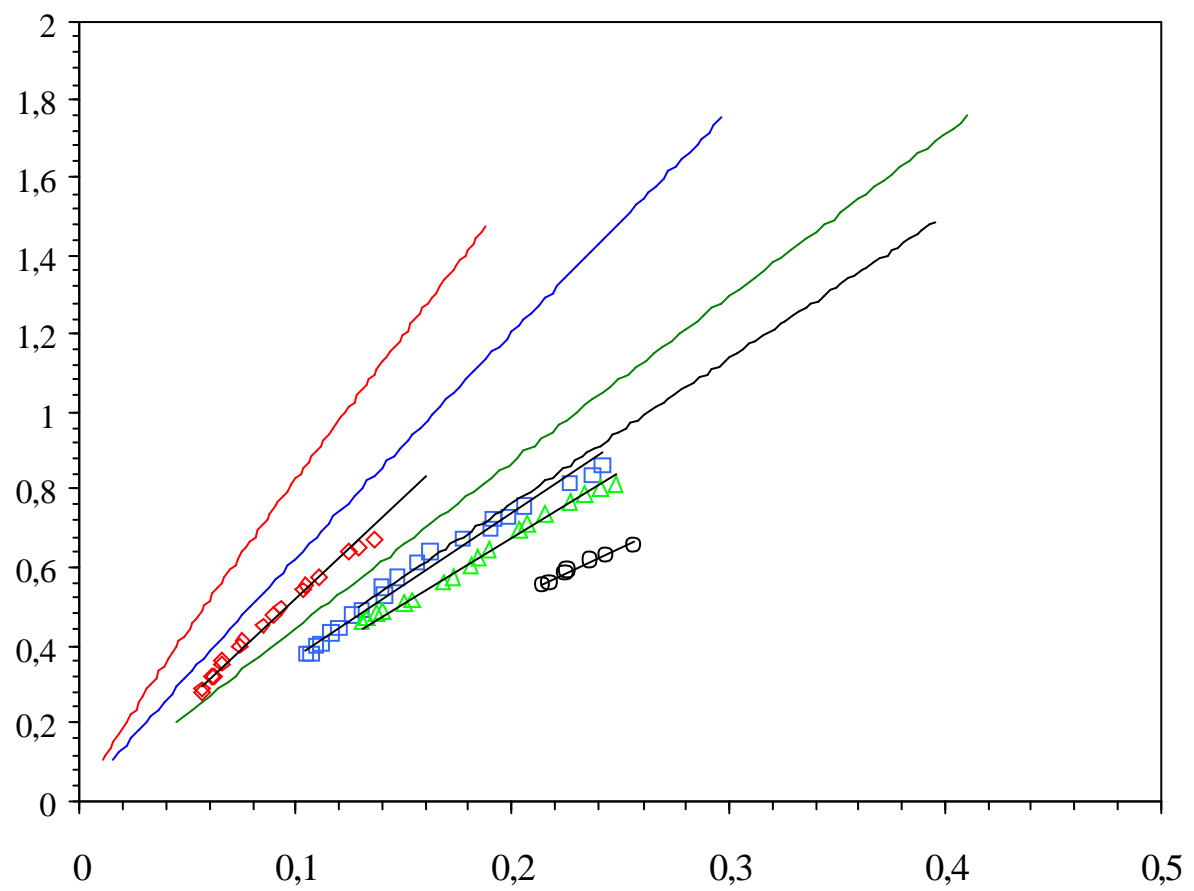

Fig.2. Ressaut contrôlé en canal à fond rugueux. Variation de la hauteur relative aval yz en fonction du débit relatif $\mathrm{Q}^{*}$, pour les quatre valeurs de $\mathrm{y}_{1}:$ ( ) 0.0939, ( ) 0.1388, ( ) 0.2122 et (0) 0.2489. ( ) Courbes d'ajustement.

L'analyse des points de mesures expérimentales du ressaut contrôlé en canal profilé en U à fond montre clairement que pour chaque valeur de $\mathrm{y}_{1}$, un ajustement de type linéaire est possible de la forme : $\mathrm{y}_{2}=\mathrm{a}_{4} \mathrm{Q}^{*}$.

Le tableau 1 regroupe les valeurs des coefficients $\mathrm{a}_{4}$

Tableau 1. Coefficients de a4 des courbes d'ajustements

\begin{tabular}{|c|c|c|c|}
\hline $\mathrm{h}_{1}(\mathrm{~cm})$ & $\mathrm{y}_{1}=\mathrm{h}_{1} / \mathrm{D}$ & $\begin{array}{c}\text { Coefficient } \\
\mathrm{a}_{4}\end{array}$ & $\mathrm{R}^{2}$ \\
\hline 2,3 & 0,0939 & 5,1977 & 0,9869 \\
3,4 & 0,1388 & - & 0,9722 \\
5,2 & 0,2122 & 3,3853 & 0,9886 \\
6,1 & 0,2490 & 2,6035 & 0,9793 \\
\hline
\end{tabular}

Le tableau 1 montre l'augmentation progressive du coefficient a avec la diminution de la hauteur relative amont $\mathrm{y}_{1}$. L'ajustement statistique des couples des valeurs ( $\left.\mathrm{y}_{1}, \mathrm{a}_{4}\right)$ par la méthode des moindres carrés donne une relation de type linéaire d'équation : 
$a_{4}=-16,43 y_{1}+6,77$. La figure 3 représente l'equation de l'ajustement.

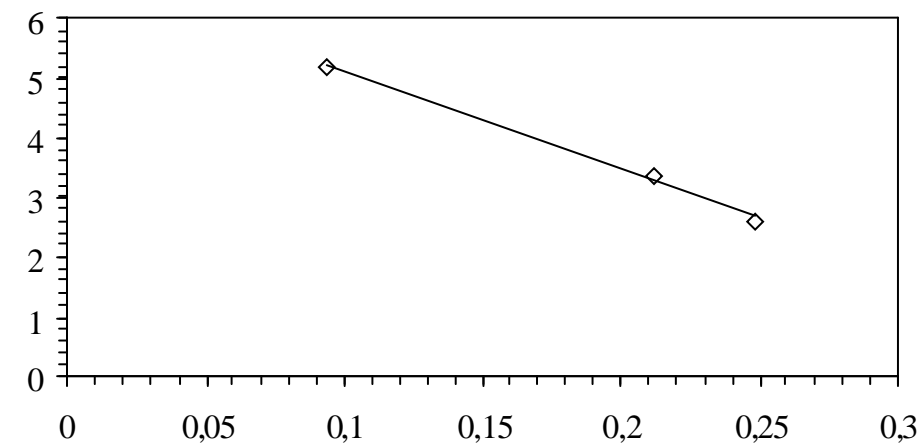

Fig.3. Variation du coefficient $\mathrm{a}_{4}$ en fonction de la hauteur amont $\mathrm{y}_{1}$

En effet, en remplaçant les coefficients a4 par son expression dans la relation $\mathrm{y}_{2}=\mathrm{a}_{4} \mathrm{Q}^{*}$, l'équation liant la hauteur relative aval $\mathrm{y}_{2}=\mathrm{h}_{2} / \mathrm{D}$ au débit relatif $\mathrm{Q}^{*}$ et à la hauteur relative amont $\mathrm{y}_{1}$ devient :

$$
\begin{aligned}
\mathrm{y}_{2}= & \left(-16,43 \mathrm{y}_{1}+6,77\right) \mathrm{Q}^{*} \\
& \operatorname{avec} 0,0939 \leq y_{1} \leq 0,2489
\end{aligned}
$$

La figure 4 montre que la relation $\mathrm{y}_{2}=\mathrm{f}\left(\mathrm{Q}^{*}, \mathrm{y}_{1}\right)$ ajuste avec une bonne corrélation les points de mesures expérimentales. Ces points suivent parfaitement la première bissectrice

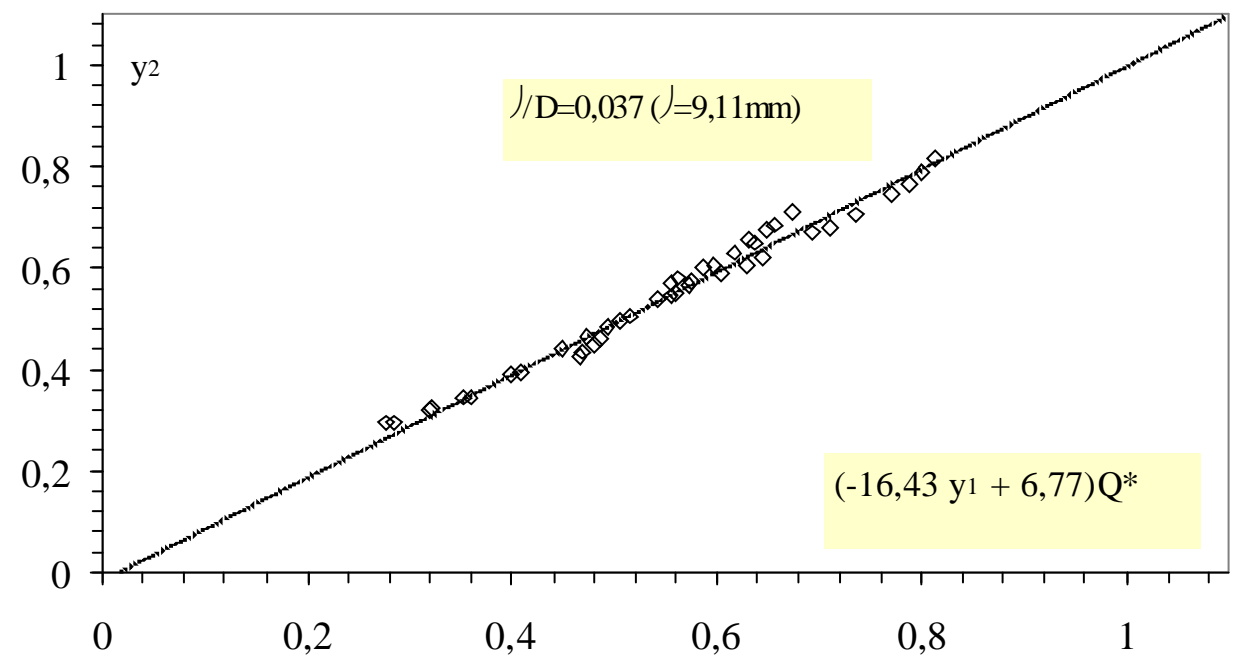

Fig.4. Variation de la hauteur relative aval $\mathrm{y}_{2}$ en fonction de ( $\left.\mathrm{y}_{1} \mathrm{Q}^{*}\right)$.( $)$ Points expérimentaux du ressaut contrôlé en canal profilé à fond rugueux de rugosité relative $/ \mathrm{D}=0,037$ ( ) Première bissectrice d'équation : $\mathrm{y}_{2}=\left(-16,43 \mathrm{y}_{1}+6,77\right) \mathrm{Q}^{*}$ 


\section{Hauteur relative aval $y_{2}=h_{2} / D$ en fonction de la hauteur relative $S=s / h_{1}$ du seuil}

La figure5 montre la représentation graphique de la variation de $\mathrm{y}_{2}$ en fonction de la hauteur relative $\mathrm{S}=\mathrm{s} / \mathrm{h}_{1} \mathrm{du}$ seuil du ressaut hydraulique contrôlé par seuil en canal à fond rugueux, de rugosité relative $\varepsilon / \mathrm{D}=0,037(\varepsilon=9,11 \mathrm{~mm})$ pour quatre valeurs de $\mathrm{y}_{1}$.

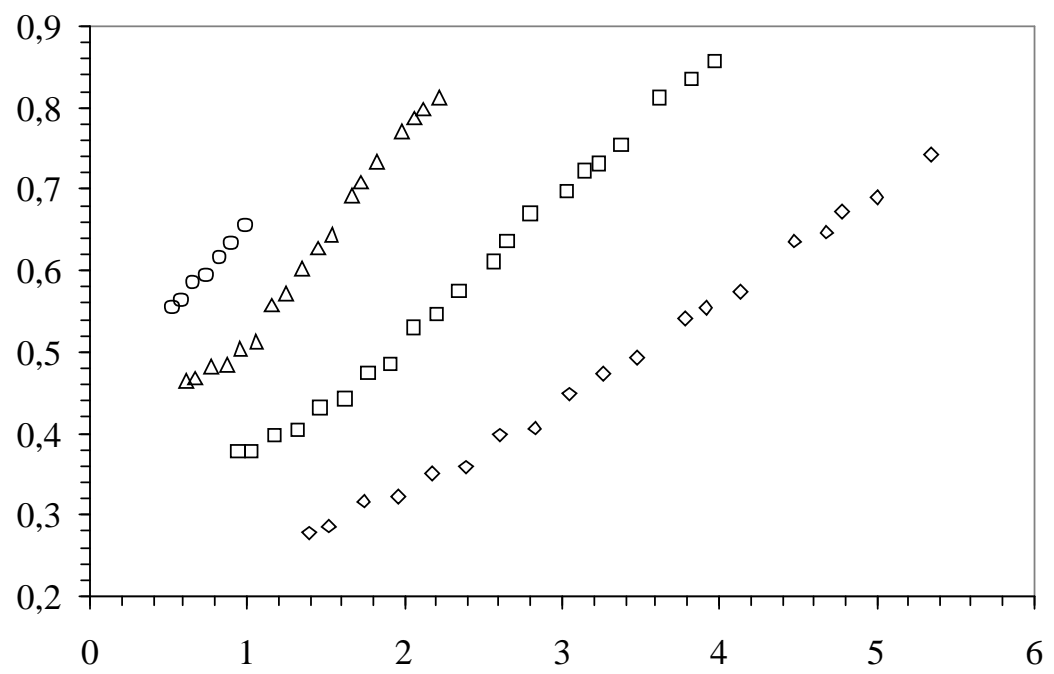

Fig.5. Variation de la hauteur relative aval $\mathrm{y}_{2}=\mathrm{h}_{2} / \mathrm{D}$ en fonction de la hauteur relative $\mathrm{S}=\mathrm{s} / \mathrm{h}_{1}$ du seuil, pour quatre valeurs distinctes de $\mathrm{y}_{1}$. Les motifs noirs vides représentent les points de mesures expérimentales.

Cette figure montre que les points de mesures du ressaut contrôlé à fond rugueux $(\varepsilon / \mathrm{D}=0,037)$ sont distincts, pour les quatre valeurs de $\mathrm{y}_{1}$. En outre, l'augmentation de la hauteur relative $S$ du seuil entraîne celle de la hauteur relative aval y2. On constate aussi que pour une même valeur de $\mathrm{S}, \mathrm{y}_{2}$ augmente avec l'augmentation de $\mathrm{y}_{1}$.L'analyse des points de mesures expérimentales du ressaut contrôlé en canal à fond rugueux, montre que pour chaque valeur de $\mathrm{y}_{1}$ une courbe de type puissance est possible de la forme $\mathrm{y}_{2}=$ $\mathrm{c}_{4} \exp \left[\mathrm{d}_{4} . \mathrm{s} / \mathrm{h}_{1}\right]$ comme le montre bien la figure 6. 


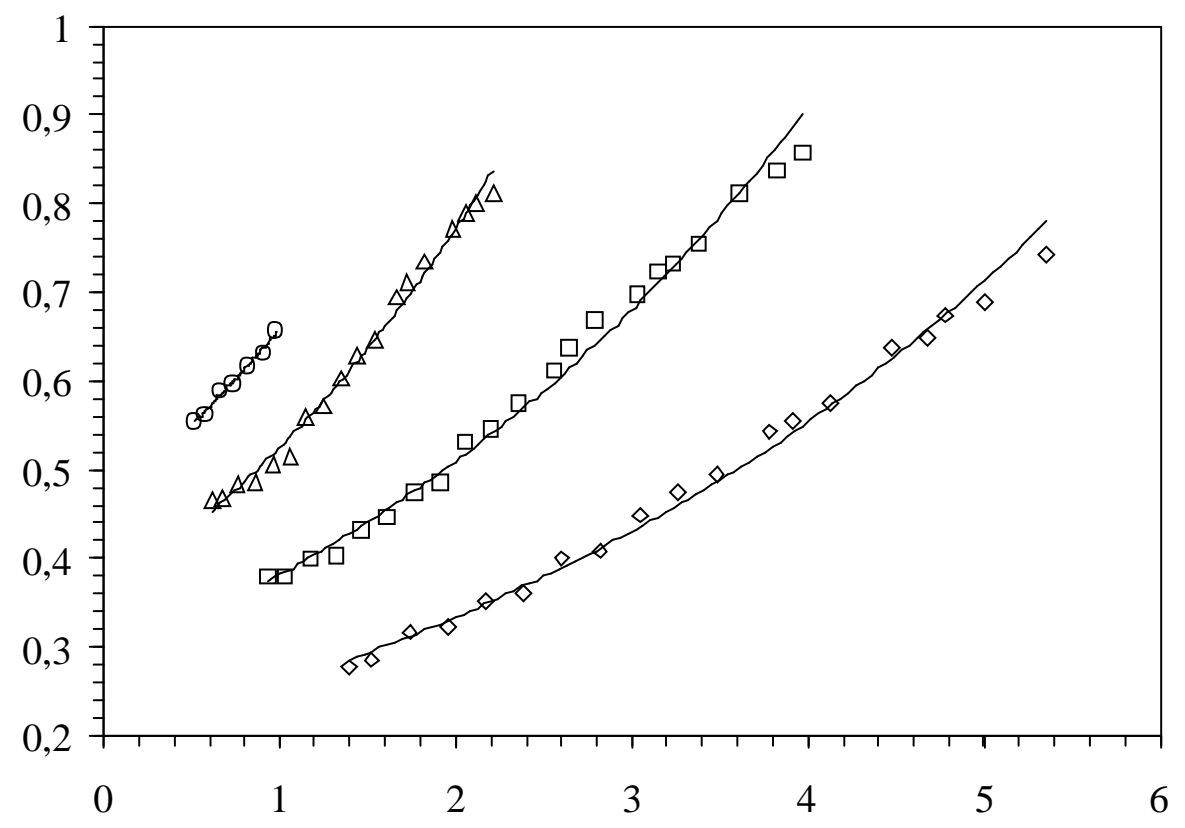

Fig.6. Ressaut contrôlé en canal profilé en U à fond rugueux. Variation de la hauteur relative aval $\mathrm{y}_{2}$ en fonction de la hauteur relative $\mathrm{S}=\mathrm{s} / \mathrm{h}_{1}$ du seuil, pour quatre valeurs différents de $\mathrm{y}_{1}:($ ) 0,0939,( ) 0,1388( ) 0,2122 et ( ) 0,2489. ( ) Courbes d'ajustements

Le tableau 2 regroupe les valeurs des coefficients $c_{4}$ et $d_{4}$.

Tableau 2. Coefficients $c_{4}$ et $d_{4}$ des courbes d'ajustements

\begin{tabular}{|c|c|c|c|c|}
\hline $\mathrm{h}_{1}(\mathrm{~cm})$ & $\mathrm{y}_{1}=\mathrm{h}_{1} / \mathrm{D}$ & $\begin{array}{c}\text { Coefficient } \\
\mathrm{c}_{4}\end{array}$ & $\begin{array}{c}\text { Coefficient } \\
\mathrm{d}_{4}\end{array}$ & $\mathrm{R}^{2}$ \\
\hline 2,3 & 0,0939 & 0,2011 & 0,2532 & 0,9930 \\
3,4 & 0,1388 & 0,2856 & 0,2893 & 0,9922 \\
5,2 & 0,2122 & 0,3572 & - & 0,9906 \\
6,1 & 0,2489 & 0,4602 & 0,3578 & 0,9919 \\
\hline
\end{tabular}

Le tableau 2 montre nettement que le coefficient $c_{4}$ augmente progressivement avec l'augmentation de la hauteur relative amont $\mathrm{y}_{1}$. L'ajustement statistique des couples de valeurs $\left(\mathrm{c}_{4}, \mathrm{y}_{1}\right)$ par la méthode des moindres carrés donne une relation de type puissance : $\mathrm{c}_{4}=1,31 \mathrm{y}_{1}{ }^{0,79}$ Celle-ci est présentée sur la figure 7 . 


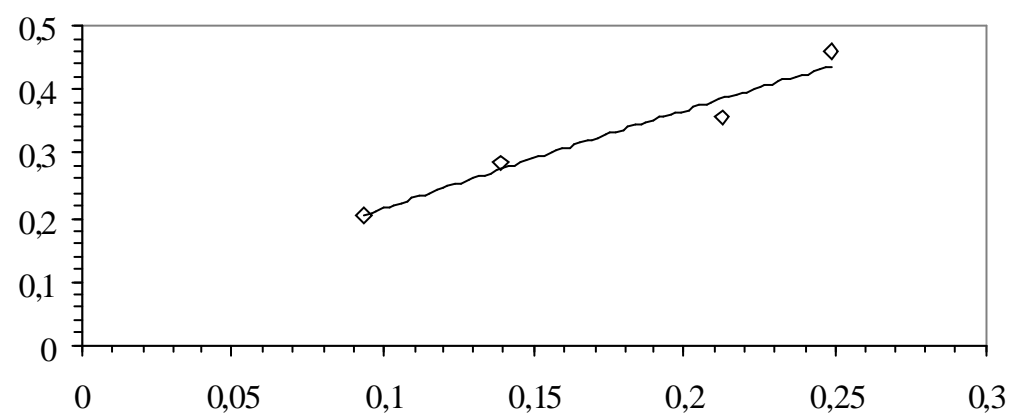

Fig.7. Variation du coefficient $\mathrm{c}_{4}$ en fonction de la hauteur relative amont $\mathrm{y}_{1}$.

Un ajustement des couples de valeurs $\left(\mathrm{d}_{4}, \mathrm{y}_{1}\right)$ du tableau 2, donne avec une bonne corrélation, la relation linéaire suivante : $\mathrm{d}_{4}=0,58 \mathrm{y}_{1}, 36$.Cette équation est montrée à la figure 8 .

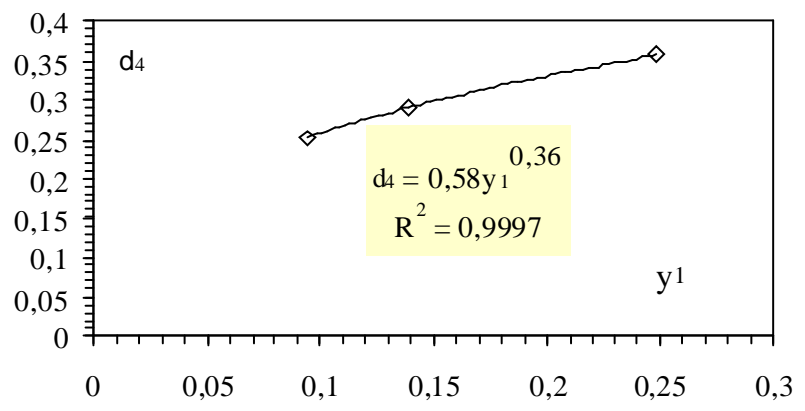

Fig.8. Variation du coefficient $\mathrm{d}_{4}$ en fonction de la hauteur relative amont $\mathrm{y}_{1}$.

En effet, en remplaçant les coefficients de $\mathrm{c}_{4}$ et $\mathrm{d}_{4}$ par leurs expressions dans la relation $\mathrm{y}_{2}=\mathrm{c}_{4} \exp \left[\mathrm{d}_{4} \cdot \mathrm{S} / \mathrm{h}_{1}\right]$, l'équation liant la hauteur relative aval $\mathrm{y}_{2}$, la hauteur relative $\mathrm{S}$ du seuil et la hauteur relative amont $\mathrm{y}_{1}$ devient :

$$
\mathrm{y}_{2}=1,31 \mathrm{y}_{1}{ }^{0,79} \exp \left[0,58 \mathrm{y}_{1}{ }^{0,36} \cdot \mathrm{s} / \mathrm{h}_{1}\right]
$$

avec $0,0939 \leq \mathrm{y}_{1} \leq 0,2489$.

La figure 9 montre ainsi que la relation trouvée $\mathrm{y}_{2}=\mathrm{f}\left(\mathrm{S}, \mathrm{y}_{1}\right)$ adapte avec une bonne corrélation les points de mesures. Ces points suivent parfaitement la première bissectrice. 


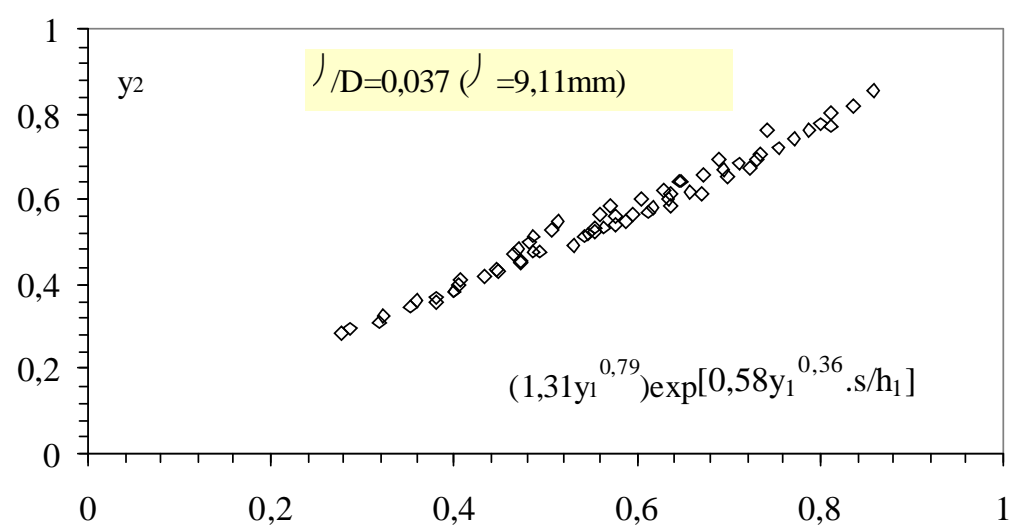

Fig.9. Variation de la hauteur relative aval $\mathrm{y}_{2}$ en fonction de $\mathrm{f}\left(\mathrm{S}, \mathrm{y}_{1}\right)$. ( )

Points de mesures expérimentales du ressaut contrôlé à fond rugueux. ( )

Première bissectrice d'équation

$$
\mathrm{y}_{2}=1,31 \mathrm{y}_{1}{ }^{0,79} \exp \left[0,58 \mathrm{y}_{1}{ }^{0,36} \cdot \mathrm{s} / \mathrm{h}_{1}\right]
$$

\section{Longueur relative $L j / h_{1}$ du ressaut en fonction du débit relatif $Q^{*}$}

La figure 10 montre la variation de la hauteur relative $\mathrm{Lj} / \mathrm{h}_{1} \mathrm{du}$ ressaut en fonction du débit relatif $\mathrm{Q}^{*}$, pour quatre valeurs différentes de la hauteur relative amont $\mathrm{y}_{1}$. Les motifs vides noirs et vides colorés représentent les points de mesures expérimentales, respectivement du ressaut 'à fond lisse' et son homologue 'à fond rugueux'. Il ressort de cette figure, que les points de mesures du ressaut 'à fond lisse' se situent bien au-dessus de ceux du ressaut 'à fond rugueux'.

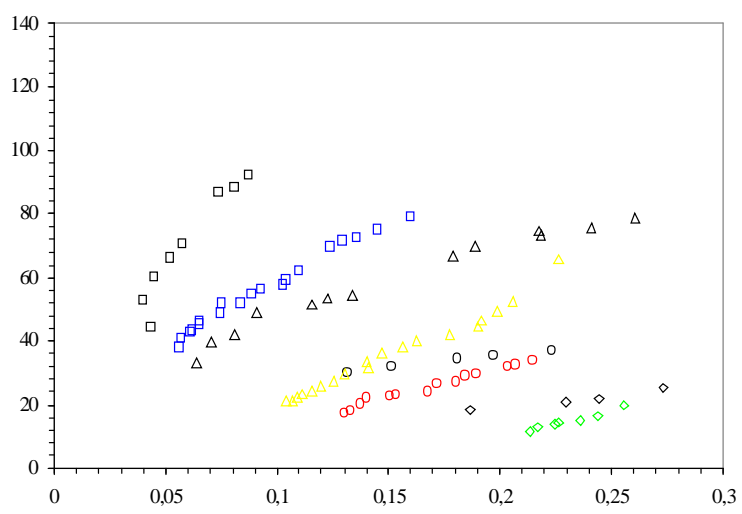

Fig.10. Variation de la longueur relative $\mathrm{Lj} / \mathrm{h}_{1}$ du ressaut en fonction du débit relatif $\mathrm{Q}^{*}$ pour quatre valeurs distinctes de $\mathrm{y}_{1}$. Les motifs colorés vides représentent les points expérimentaux du ressaut contrôlé en canal à fond rugueux. Les motifs vides noirs représentent le ressaut contrôléà fond lisse. 
Nous pouvons conclure que pour $0,0939 \leq \mathrm{y}_{1} \leq 0,2489$, la longueur du ressaut contrôlé en canal à fond rugueux est plus réduite que celle de son homologue à fond lisse. Par conséquent l'effet de réduction de la longueur du ressaut est perceptible.

\section{CONCLUSION}

Dans cette étude, l'effet de la rugosité absolue $\varepsilon=9,11 \mathrm{~mm}$ imposé au fond du canal profilé en " U ", de pente horizontal, sur les caractéristiques du ressaut hydraulique a été justifié.

Dans un premier temps, l'étude s'est intéressée à la variation de la hauteur relative aval $\mathrm{h}_{2} / \mathrm{D}$ du ressaut en fonction du débit relatif $\mathrm{Q}^{*}$ de l'écoulement incident pour quatre ouverture différentes. Il a été constaté que pour une valeur du débit relatif $\mathrm{Q}^{*}$, l'augmentation de la rugosité absolue entraîne la diminution de la hauteur relative aval .par ailleurs, l'analyse statistique des points de mesures expérimentales a montré que, pour une rugosité absolue testée, la hauteur relatif aval $h_{2} / D$ était lié au débit relatif $Q^{*}$ par la loi de type linéaire [Equation 1 ].

Dans un second temps, l'étude a traité la variation de la hauteur relative aval $\mathrm{h}_{2} / \mathrm{D}$ du ressaut en fonction de la hauteur relative du seuil $\mathrm{S}=\mathrm{s} / \mathrm{h}_{1}$ pour quatre ouvertures distinctes. Il a été remarqué que pour l'augmentation de la hauteur relative $S$ du seuil entraîne celle de la hauteur relative aval y2. On observe aussi que pour une même valeur de $S_{1} y_{2}$ augmente avec l'augmentation de $\mathrm{y}_{1}$.

En outre, l'analyse statistique des points de mesures expérimentales a montré que, pour cette rugosité testée, la hauteur relatif aval $\mathrm{h}_{2} / \mathrm{D}$ était lié à la hauteur relative du seuil par la loi de type exponentielle [Equation 2].

Dans un troisième temps, l'étude s'est intéressée à la variation de la longueur relative $\mathrm{Lj} / \mathrm{h}_{1} \mathrm{du}$ ressaut en fonction du débit relatif $\mathrm{Q}^{*}$ de l'écoulement incident pour quatre valeurs distinctes de la hauteur relative amont $\mathrm{y}_{1}=\mathrm{h}_{1} / \mathrm{D}$.Les motifs vides noirs et vides colorés représentent les points de mesures expérimentales, respectivement du ressaut, à fond lisse' et à fond rugueux'. Il ressort de cette figure, que les points de mesures du ressaut 'à fond lisse' se situent nettement au-dessus de ceux du ressaut 'à fond rugueux'. 
Enfin, on peut conclure, que pour une série des débits relatif $\mathrm{Q}^{*}$, le ressaut hydraulique évoluant dans un canal profilé en ' $U$ ' à fond rugueux, présente un effet réducteur de la longueur de ressaut et une hauteur aval inférieur que son homologue à fond lisse.

\section{PRINCIPALES NOTATIONS}

\begin{tabular}{|c|c|c|}
\hline $\mathrm{D}$ & Diamètres, largeur du canal & [m] \\
\hline $\mathrm{g}$ & Accélération de la pesanteur $\left[\mathrm{m} / \mathrm{s}^{2}\right]$ & \\
\hline $\mathrm{h}_{1}$ & Hauteur initiale du ressaut & [m] \\
\hline $\mathrm{h}_{2}$ & Hauteur finale du ressaut & $\mathrm{n}]$ \\
\hline $\mathrm{Lj}$ & Longueur du ressaut & \\
\hline $\mathrm{Lr}$ & Longueur du rouleau & $\mathrm{n}$ \\
\hline $\mathrm{Lj}^{*}$ & Longueur du ressaut classique & \\
\hline $\mathrm{P}$ & Pression $\left[\mathrm{N} / \mathrm{m}^{2}\right]$ & \\
\hline Q & Débit volume $\left[\mathrm{m}^{3} / \mathrm{s}\right]$ & \\
\hline $\mathrm{Q}^{*}$ & Débit relatif & [-] \\
\hline $\mathrm{R}_{\mathrm{h}}$ & Rayon hydraulique & \\
\hline $\mathrm{IRe}$ & Nombre de Reynolds & \\
\hline $\mathrm{s}$ & Hauteur d'un seuil & \\
\hline $\mathrm{S}$ & Hauteur relative d'un seuil & \\
\hline \multirow[t]{2}{*}{$\mathrm{V}$} & Vitesse d'écoulement & \\
\hline & $\mathrm{s}]$ & \\
\hline $\mathrm{x}$ & Coordonnée longitudinale & \\
\hline $\mathrm{y}_{1}$ & hauteur relative amont du ressaut $\left(h_{1} / D\right)$ & \\
\hline $\mathrm{y}_{2}$ & hauteur relative aval du ressaut $\left(h_{2} / D\right)$ & \\
\hline \multirow[t]{2}{*}{$\varepsilon$} & Rugosité équivalente & \\
\hline & $\mathrm{m}]$ & \\
\hline$\rho$ & Masse volumique & \\
\hline$\varpi$ & Poids spécifique $\left[\mathrm{N} / \mathrm{m}^{3}\right]$ & \\
\hline
\end{tabular}

\section{REFERENCES}

[1] Achour B., Debabeche M., Ressaut contrôlé par seuil dans un canal profile en $U$. Journal of hydraulic research.2003, 46, 97-103

[2] Debabeche M., Achour B., Ressaut hydraulique classique en canal profilé en ' $U$ '. $1^{\mathrm{ER}}$ Séminaire Sur L’eau, Université de Mascara, 2000.

[3] Debabeche M., Kateb S., Ghomri A., Etude du Ressaut Hydraulique dans un canal triangulaire à parois rugueuses. Journal of hydraulic research.2006, 05,187-196

[4] Ghomri A.,Debabeche M., Riguet F., Etude expérimentale évoluant dans un canal profilé en $\mathrm{U}$, à fond rugueux. Revues des sciences fondamentales et 
appliquées.2009,01,80-103

[4] Ghomri A.,Debabeche M., Riguet F., L'etude Semi-théorique du ressaut hydraulique évoluant dans un canal profilé en $\mathrm{U}$, à fond rugueux . Revues des sciences fondamentales et appliquées.2009,01,41-56

[5] Hager W.H, Der Ablufss im U-Profil. Korrespondenz Abwasser, 1987, 34(5), 468-482.

[6] Hager W.H., Hydraulic jump in U-channel, Proc. ASEC, J. Hydraulic Engineering $.1989,115,667-675$

[7] Rajartnam N., Hydraulic jumps on rough beds, Transaction of the engineering institute of Canada, 1968, 11, № A-2,

[8]Rand W., An approach to generalized design of stilling basins, Trans. New York Academy of Sciences, 1957, 20 (2), 173-191.

[9] Achour B. (1998) Dissipation d'énergie par ressaut, thèse de doctorat, Université de Tizi-Ouzou.

[10] Debabeche M. (2003).Ressaut hydraulique dans les canaux prismatiques. Thèse de doctorat d'état, Université de Biskra. 


\section{EFFET D'UNE RUGOSITE AU RESSAUT HYDRAULIQUE DANS UN CANAL PROFILE EN "U" A FOND RUGUEUX}

RESUME Cette étude a pour objectif d'étudier le ressaut hydraulique contrôlé par seuil, évoluant dans un canal profilé en ' $U$ ' à fond rugueux pour une seule rugosité. Des relations fonctionnelles, en termes adimensionnels, liant les différentes caractéristiques du ressaut, faisant apparaître l'effet de la rugosité du fond du canal, sont obtenues.

Mots clés : Ressaut hydraulique, rugosité, bassin d'amortisseur, canal à fond rugueux.

How to cite this article

Ghomri A, Debabeche M and Riguet F. Effect of roughness to hydraulic projection in an U-shaped channel, with rough bed. J Fundam Appl Sci. 2011, 3(1), 94-110. 